Pathologe 2009 • [Suppl 2] 30:252

DOI 10.1007/s00292-009-1245-x

(C) Springer Medizin Verlag 2009

Roland Pauli

Institut für Pathologie, Brandenburg an der Havel

\title{
Hansjürgen Zschoch
}

\subsubsection{0-07.04.2009}

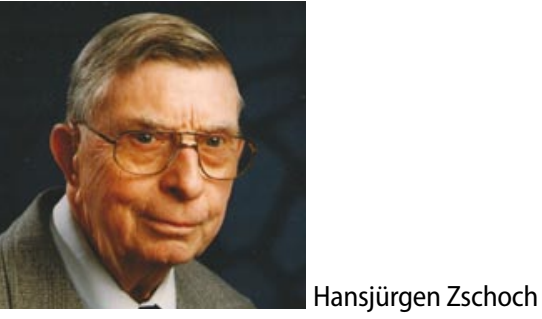

Hansjürgen Zschoch wurde am 29. August 1930 geboren. Er war der Sohn eines niedergelassenen HNO-Arztes in Liegnitz (Schlesien). Bedingt durch den Krieg verließ er zusammen mit seinen Großeltern und seiner jüngeren Schwester die Heimatstadt in Richtung Sachsen. Seine Eltern kamen auf dem Weg dorthin, am 13. Februar 1945 bei dem schweren Bombenangriff auf Dresden ums Leben. Zufluchtsort wurde ihm Chemnitz, wo er 1949 das Abitur ablegte.

Hansjürgen Zschoch studierte und promovierte an der Universität Leipzig. 1955 begann er an gleicher Stelle eine Ausbildung zum Pathologen. $\mathrm{Zu}$ seinen Lehrern gehörten Heinrich Bredt und ab 1959 Gottfried Holle, unter deren fruchtbarem Einfluss (Leipziger Schule) mehrere bedeutende Pathologen hervorgingen.

1960 erwarb Hansjürgen Zschoch die Facharztanerkennung für Pathologische Anatomie. Er blieb an der Leipziger Universität, wo er sich in Oberarztfunktion 1965 habilitierte („Die Herz- und Gefäßerkrankungen in der Sektionsstatistik"). Im gleichen Jahr übernahm er die Chefarztstelle am Pathologischen Institut des Bezirkskrankenhauses Brandenburg/ Havel, die er bis 1989 inne hatte. In den Jahren seiner Amtszeit in der Stadt Brandenburg hat Hansjürgen Zschoch unter den nicht einfachen Rahmenbedingungen der DDR nicht nur viel für die Entwick- lung des Fachgebietes Pathologie getan. Er war zugleich ein vielgefragter Ratgeber der Kliniker, Referent in zahlreichen Fortbildungsveranstaltungen und Betreuer wissenschaftlicher Arbeiten (2 Habilitationen, 31 medizinische Dissertationen und 3 Diplomarbeiten). Im damaligen Bezirk Potsdam war er in mehreren Kommissionen des Gesundheitswesens tätig. Als Vorsitzender des Literaturbeirates nahm er maßgeblichen Einfluss auf den Erwerb von medizinischen Fachbüchern und -zeitschriften aus westlichen Ländern.

Hansjürgen Zschoch war Mitglied der Deutschen Gesellschaft für Pathologie.

Es war immer sein persönliches Anliegen, seinen Schülern fachliches Wissen frei von gesellschaftspolitischen Zwängen zu vermitteln.

Sein wissenschaftliches Interesse galt insbesondere der vergleichenden Pathologischen Anatomie, wovon über 200 Publikationen in Zeitschriften sowie zahlreiche Buchbeiträge zeugen. Die letzten Berufsund Lebensjahre waren überschattet von schweren Erkrankungen, denen er jedoch mit bewundernswertem Willen trotzte.

Mit Hansjürgen Zschoch verlieren wir einenfachlichundmenschlich hervorragenden Vertreter des Fachgebietes Pathologie und einen hochangesehenen Mentor und Förderer der Ärzteschaft in der Region.

Er hinterlässt seine Ehefrau Lore, mit der er über 50 Jahre verbunden war.

\section{Roland Pauli, Brandenburg}

\section{Korrespondenzadresse \\ Dr. Roland Pauli \\ Institut für Pathologie, \\ Gesundheitszentrum Brandenburg an der Havel Hochstraße 29 \\ 14770 Brandenburg \\ pauli@klinikum-brandenburg.de}

